\title{
Effects of Slip Planes on Stresses in MICE Coupling Solenoid Coil Assembly
}

\author{
L. Wang, H. Pan , H. Wu, X. L. Guo, Y, Cheng, and M. A. Green, Member, IEEE
}

\begin{abstract}
The MICE superconducting coupling solenoid magnet is made from copper matrix $\mathrm{Nb}$ - $\mathrm{Ti}$ conductors with inner radius of $750 \mathrm{~mm}$, length of $285 \mathrm{~mm}$ and thickness of $110.4 \mathrm{~mm}$ at room temperature. The coil is to be wound on a mandrel made of aluminum. The peak magnetic field on the conductor is about 7.3 $\mathrm{T}$ when fully charged at $210 \mathrm{~A}$. High magnetic field and large size make the stress inside the coupling coil assembly relatively high during cool down and full energizing. The shear stress between coil winding and aluminum casing may cause premature quench. To avoid quench potential induced by stress, slip planes were designed for the coil assembly. In this paper, FE models with and without slip planes for it have been developed to simulate the stresses during the process including winding, cooling down and charging. The stress distribution in the coil assembly with and without slip planes was investigated. The results show that slip planes with low friction coefficients can improve the stress condition in the coil, especially reduce the shear stress largely so that improve the stability.
\end{abstract}

Index Terms-Friction coefficient, MICE superconducting coupling magnet, Slip planes, Stress analysis,

\section{INTRODUCTION}

$\mathrm{T}$ HE Muon Ionization Cooling Experiment (MICE) will demonstrate ionization cooling in a short section of a realistic cooling channel using a muon beam at Rutherford Appleton Laboratory in the UK [1]. A pair of coupling solenoid magnets is used to produce up to $2.6 \mathrm{~T}$ on the magnet centerline to keep the muon beam within the iris of thin RF cavity beryllium windows [2],[3].

The coupling solenoid has an inner radius of $750 \mathrm{~mm}$, length of $285 \mathrm{~mm}$ and thickness of $110.4 \mathrm{~mm}$ at room temperature. The peak field generated by it is up to $7.3 \mathrm{~T}$ at the worst operation case of the MICE cooling channel. The stresses induced inside the coil are relatively high because of large diameter and high magnetic field [4],[5]. The shear stress is principally responsible for premature quench, which might

Manuscript received 20 October 2009. This work was supported by Funds of cryogenics and superconductivity technology innovation project under " $985-2$ Plan" of Harbin Institute of Technology, China and by the Office of Science, US Department of Energy under DOE contract DE-AC02-05CH11231.

L. Wang was with the Institute of Cryogenics and Superconductivity Technology, HIT, Harbin 150001, China. She is now with Shanghai Institute of Applied Physics, Shanghai 201204, China (phone: 86-21-33932552, e-mail: wangli_icst@hit.edu.cn).

H. Pan, H. Wu, X. L. Guo, Y. Cheng are with the Institute of Cryogenics and Superconductivity Technology, Harbin Institute of Technology, Harbin 150001, China. (panheng@hit.edu.cn)

M. A. Green is with Lawrence Berkeley National Laboratory, Berkeley, CA, 94720 USA (e-mail: magreen@lbl.gov). cause fracture of epoxy resin in the coil winding and then part of the strain energy to convert into heat [6]. Slip planes in a superconducting magnet, which allow the coil package to slide relative to the mandrel, are helpful to change the contact conditions so as to reduce the shear stress [7].

The finite element models with and without slip planes in the coil assembly have been developed to investigate the effects of slip planes on stress and stability of the MICE coupling magnet in this paper. The stress distribution within the coupling coil assembly with different contact conditions was analyzed.

\section{The MiCE Coupling CoIL Assembly AND Finite ELEMENT MODELS}

\section{A. The MICE Coupling Coil Assembly}

The MICE coupling coil is a single solenoid wound on a 6061-T6-Al mandrel at a constant winding pre-stress of $60 \mathrm{MPa}$ [5].

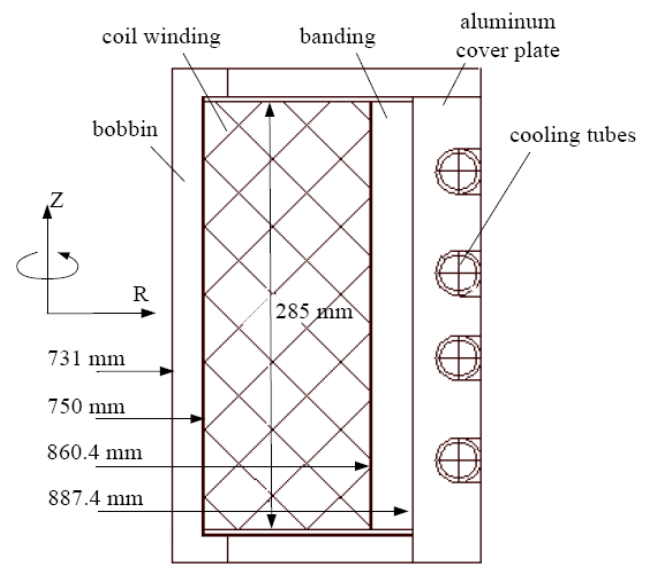

Fig. 1. The cross-section of MICE coupling coil assembly

The 2-D view of the MICE coupling coil assembly is shown in Fig. 1. The coil assembly comprises the coupling coil, the banding and the coil mandrel. The coupling coil is composed of fiberglass cloth and thin G-10 plates for electrical insulations, Stycast epoxy resin, and copper stabilized NbTi conductors. Outside the outermost layer of the coil winding is the banding made of aluminum alloy with the thickness of $27 \mathrm{~mm}$. The mandrel made of 6061-T6 aluminum includes a bobbin, two end plates and a cover plate, which are $19 \mathrm{~mm}, 18 \mathrm{~mm}$ and 45 $\mathrm{mm}$ thick, respectively. The cooling cryogen flows in the tubing nested into the cover plate to cool the coil by heat conduction. 


\section{B. The Finite Element Model}

Two kinds of 2-D axial symmetric models for the coupling coil assembly separately with and without slip planes were developed to study the effects of slip planes on stresses. Both of the models include the coil, the mandrel, and the banding. The coupling coil has 96 layers. In order to simulate the winding pre-stress in the coil exactly, the coil was subdivided into 96 layers and the banding was sub-divided into 6 layers in the radial direction.

In the model without slip planes, all of the components are glued together in the simulation. By comparison, the components are not bonded in the model with slip planes but contact elements are adopted. They are allowed to interact via contact elements. The contact element for simulating slip planes solved the control equations of finite elements by handling the two problems: the normal contact and tangent sliding. The control equations of contact elements in the two contact surfaces are [8]:

$\left[\mathbf{K}_{i}\right]\left\{\mathbf{U}_{i}\right\}=\left[\mathbf{F}_{i}\right]+\left[\mathbf{R}_{i}\right], i=1,2$

where $\boldsymbol{K}$ is the stiffness matrix of elements; $\boldsymbol{U}$ is the displacement of nodes; $\boldsymbol{F}$ is the external loads; $\boldsymbol{R}$ is the contact force matrix. The slip model adopted the Augmented Lagrange method and Coulomb friction law to calculate the normal contact force and tangent friction force, respectively.

The contact elements are used in three places: one is on the interface of the coil and the insulated bobbin; the other two are on interfaces of $0.5 \mathrm{~mm}$ thick side G-10 plates adhered to the mandrel end plates and $3 \mathrm{~mm}$ thick side $\mathrm{G}-10$ plates glued with the coil as shown in Fig. 1. Different frictional sliding conditions have been considered for all the contact surfaces between the coil package and the surrounding components.

When winding the coil, the SC conductors are wound layer by layer onto the 6061-T6 Al mandrel under certain appropriate constant pre-tension, and outside the outmost layer insulated by thin G-10 plates. The banding is then wound under certain pre-stress. The 6061-T6 Al cover plate is welded onto the mandrel finally. During a normal operation, the magnet is cooled down from room temperature to $4.2 \mathrm{~K}$, firstly, and then charged to a full current of $210 \mathrm{~A}$. The models adopted the method of killing and activating element to simulate the winding process, and employed the multi-load technology to complete the sequential simulation involving the winding, cooling and charging.

The following assumptions were applied for the simulation: (1) neglecting the effect of the winding support of the mandrel for the winding process; (2) assuming the coil current centre in the axial direction to locate at original position; (3) neglecting the effect from cold mass supports; (4) assuming the coil assembly at uniform temperature of $4.2 \mathrm{~K}$.

Table I lists the material properties at room temperature used in the FE models. The elastic modulus in the radial, hoop and axial directions are $E_{r}, E_{\theta}$ and $E_{z}$, respectively. The Poisson ratios in the corresponding directions are $v_{r} v_{\theta z}$ and $v_{r z}$, respectively. The mean integral thermal expansion coefficient is $\alpha$, which is expressed as [9]:
$\alpha=\frac{\int_{T_{r e f}}^{t} \alpha(t) \mathrm{d} t}{t-T_{\text {ref }}}$

where $\alpha(t)$ is the instantaneous coefficients of thermal expansion. $T_{\text {ref }}$ is the reference temperature of $300 \mathrm{~K}$.

TABLE I. Material Properties

\begin{tabular}{ccccc}
\hline \hline Property & Coil package & G-10 & banding & mandrel \\
\hline$E_{r}(\mathrm{GPa})$ & 70 & & & \\
$E_{\theta}(\mathrm{GPa})$ & 50 & 22 & 71 & 77 \\
$E_{z}(\mathrm{GPa})$ & 90 & & & \\
$v_{r \theta}$ & 0.34 & & & \\
$v_{\theta z}$ & 0.32 & 0.2 & 0.32 & 0.31 \\
$v_{r z}$ & 0.2 & & & \\
$\alpha\left(\mathrm{K}^{-1}\right)$ & $1.149 \times 10^{-5}$ & $0.97 \times 10^{-5}$ & $1.41 \times 10^{-5}$ & $1.42 \times 10^{-5}$ \\
\hline \hline
\end{tabular}

\section{StResses With DifFERENT SLIP Planes}

The FE simulation was performed by general finite element software [9]. The pre-tensions applied to the conductors and the banding wires during winding are both $60 \mathrm{MPa}$, which result in an initial stress distribution in the coil assembly. The slip plane has little effect on the stress distribution because it mainly influences the stress in the axial and the transverse planes. The winding tension forces the coil in the radial direction. This paper primarily focused on the thermally and magnetically induced stresses within the coil with and without slip planes.

\section{A. Stresses Induced by Thermal Contraction}

The cooling down of the magnet from room temperature of $300 \mathrm{~K}$ to $4 \mathrm{~K}$ causes all the components in the coil assembly to shrink in all directions. The difference of the thermal contraction property of materials leads to an additional thermal stress. The stresses in the coil assembly after cooling are the combination of the stress after winding and the thermal stress. Fig. 2 shows the stress distribution along the mid-plane of the coil assembly with and without slip planes after cooling down from $300 \mathrm{~K}$ to $4 \mathrm{~K}$.

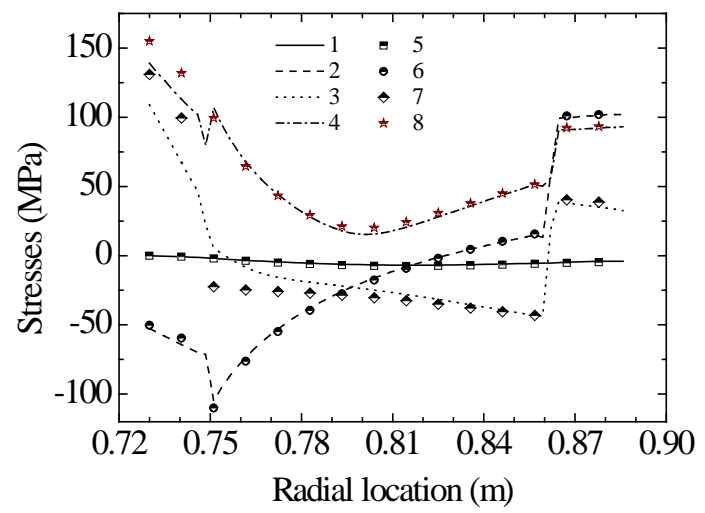

Fig. 2. The stresses along the mid-plane of the coil assembly at $4.2 \mathrm{~K}$ with and without slip planes 
The data symbols in Fig. 2 have the meaning as follows: "1-4" mean radial stress, hoop stress, cumulative axial stress and Von Mises stress of the slip model with the friction factor of 0.1 in both side and bottom slip planes; "5-8" represent radial stress, hoop stress, cumulative axial stress and Von Mises stress of the non-slip model. Fig. 3 shows the shear stress at one end of the coil with and without slip planes.

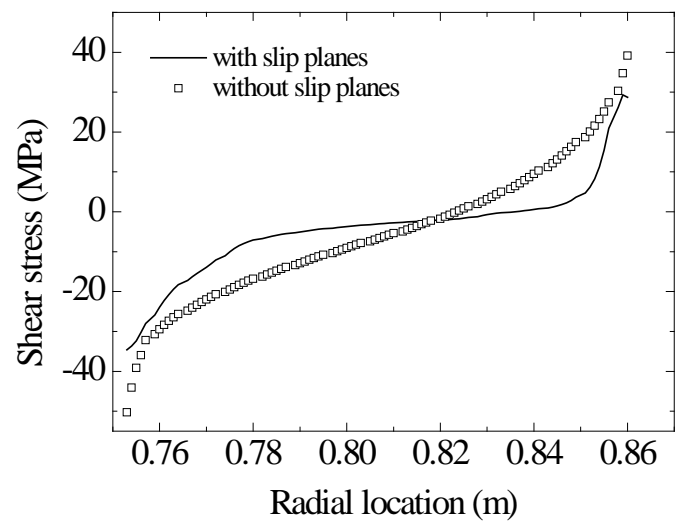

Fig. 3. The shear stress at the coil end at $4.2 \mathrm{~K}$ with and without slip planes

From Fig. 2, the stress results with slip planes are different from those without slip planes in the bobbin and the inner layers of the coil. The results of the two models approximate in the region far from the coil inner layers. The difference of Von Mises stress is $15 \mathrm{MPa}$ in the middle of the bobbin. The difference of cumulative axial stress in the coil is $25 \mathrm{MPa}$. The differences of radial stress and hoop stress are very small because the slip plane can only affect the axial stress component and the stress in the meridian plane of the coil.

From Fig. 3, the slip plane has a greater effect on the shear stress at the coil ends than the other stress components. Compared with the peak shear stress of 55 MPa without slip planes, the maximum shear stress with slip planes is nearly half of that, about $26 \mathrm{MPa}$. It indicates the slip plane mainly reduces the shear stress in the coil package.

Fig. 4 shows the relationship of the peak shear stress within the coil package and the friction coefficient of slip planes. A lower friction coefficient means a lower restriction between the coil and the mandrel. In other words, the coil is more "free".

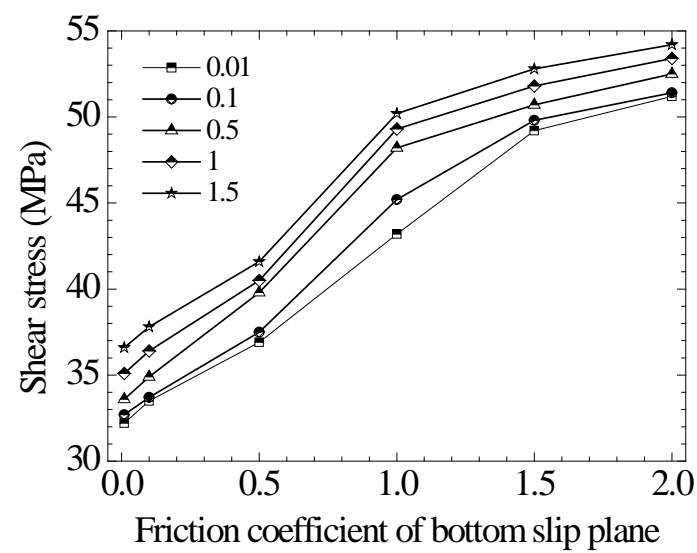

Fig. 4. The shear stress as a function of friction coefficient of bottom slip plane with different side slip planes friction coefficients.
As the friction coefficient increases, the peak shear stress increases unsteadily. "Stick slip" friction will more likely occur in the interfaces with sudden releasing energy with a larger friction coefficient. When the bottom friction coefficient exceeds 1.0, the growth rate slows down and then the stress will become like the status without slip planes. The results indicated the bottom slip plane has a greater effect on the peak shear stress than the sides slip planes. So, decreasing the friction coefficient of the bottom slip plane will release the shear stress in the coil obviously after cooling down to $4.2 \mathrm{~K}$.

\section{B. Stresses Induced by Magnetic Load}

When excited to the full current of $210 \mathrm{~A}$, the interaction of the current and the magnetic field produces a non-uniform spatial variation Lorentz body force. The Lorentz force compresses the coil in axial direction, and pushes the coil outward in radial direction. The magnetic force is the largest load in the coil package. Fig. 5 shows the contours of the magnetic field of the MICE coupling coil.

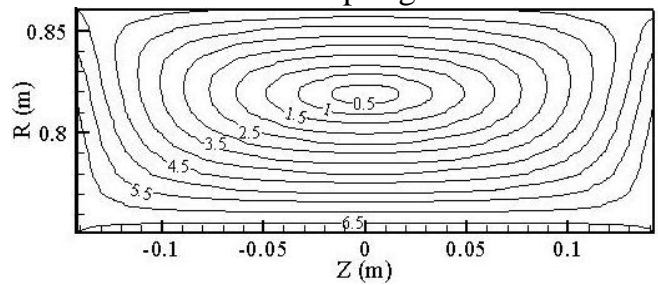

Fig. 5. The contours of the magnetic field (T) in the MICE coupling coil.

Fig. 6 shows the stresses in the coil assembly with and without slip planes when the coil is charged to $210 \mathrm{~A}$. The data symbols in Fig. 6 have the same meanings as in Fig. 2. By comparison with the stress distribution after cooling, the high stress region after energizing changed to the outer layers of the coil and the banding. The difference of the results with and without slip planes after charging shows a similar trend like the stress distribution after cooling down: in the bobbin and the inner layers of the coil, the difference is noticeable, but in the central and outer layers, the difference is unobvious. The Von Mises stress in the middle of the bobbin decreases $55 \mathrm{MPa}$ without slip planes and $45 \mathrm{MPa}$ with slip planes because of the magnetic load. The difference of the radial stress and the hoop stress is also very small.

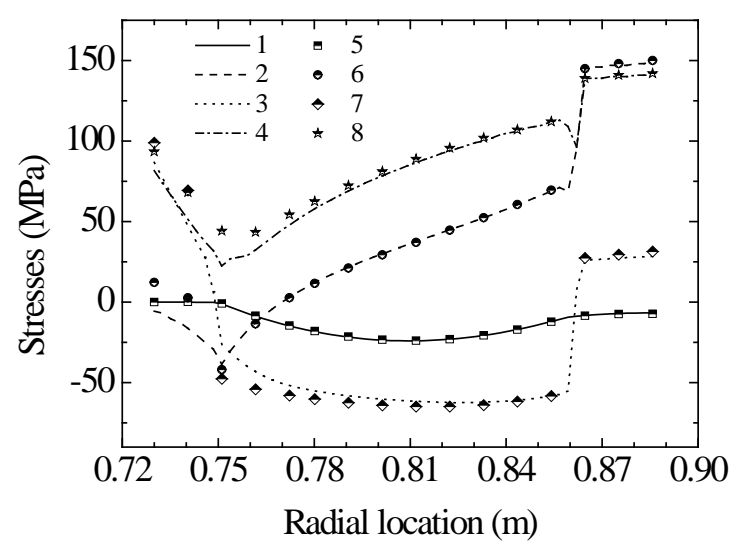

Fig. 6. The stresses in the mid-plane of the coil assembly when charged to 210 A with and without slip planes 
Fig. 7 shows the shear stress at the end of the coil with and without slip planes after charging. In the inner corner of the coil, the peak shear stress reduces both about $10 \mathrm{MPa}$ with and without slip planes under the electromagnetic loads. It shows the slip planes have a relatively minor influence on the shear stress induced by the magnetic load compared with thermal load. Slip planes mainly decreases the shear stress induced by the thermal contraction.

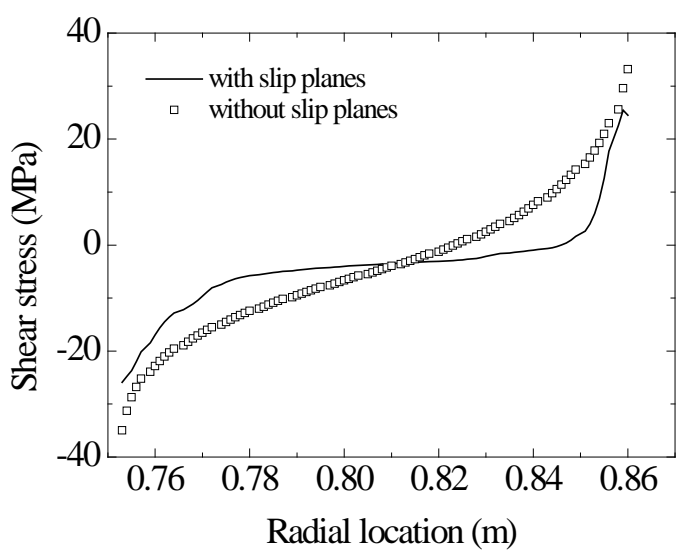

Fig. 7. The shear stress at the coil end when charged to $210 \mathrm{~A}$ with and without slip planes

Fig. 8 shows the relationship of the peak shear stress within the coil package and the friction coefficient of slip planes. The magnetic load decreases the peak shear stress within the coil package. The decrease of the peak shear stress after charging by the slip plane is small because the slip plane affects little on the shear stress caused by the magnetic load. The friction coefficient of the side slip plane has a less effect on the stress than that of the bottom slip plane.

From Fig. 4 and Fig. 8, the peak shear stress will reach the stress level without slip planes when the friction coefficients exceed 1.5. The shear strength of the epoxy resin used in the MICE coupling magnet is about $5000 \mathrm{psi}$ (34MPa) [10]. So, the friction coefficient of the slip planes to be used is better less than 0.5 .

According to the above simulation results and analyses, the following design in the MICE coupling magnet is to be adopted: two layers of 50 micrometer Kapton films are to be applied as

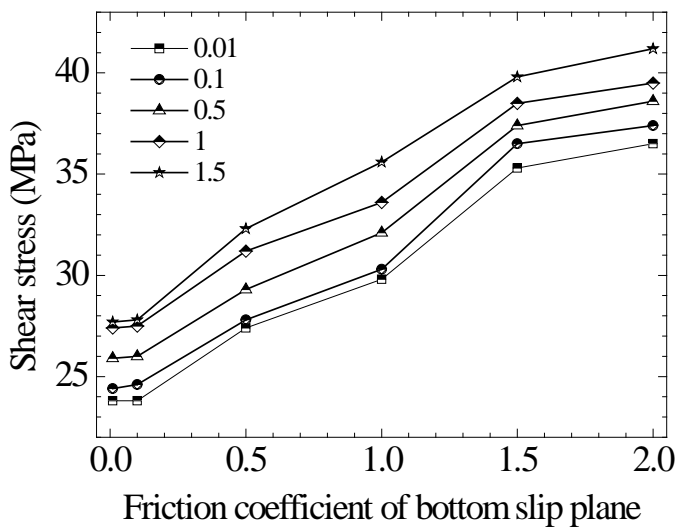

Fig. 8. The shear stress as a function of friction coefficient of bottom slip planes with different side slip plane friction coefficients. the bottom slip plane between the coil and G-10 plate insulated bobbin; The insulations between the coil and the mandrel end plates will be realized using a $0.5 \mathrm{~mm}$ G-10 sheet and a $3 \mathrm{~mm}$ G-10 plate.

\section{CONCLUSION}

The stress distribution in the MICE coupling coil with and without slip planes, respectively and effects of friction coefficient of slip planes on them were studied in detail. The slip planes mainly have a great effect on the transverse shear stresses induced by the thermal contraction during coil cool down process. It is found that when the friction coefficient of the slip plane exceeds 0.5 , the peak shear stress increases rapidly and shows obvious "stick slip" friction. The slip planes with low friction coefficient decreased the shear stresses remarkably, which may reduce the possibility of quench resulting from crack of epoxy resin inside the coil assembly.

\section{ACKNOWLEDGMENT}

The authors would like to thank the experts CHEN Hao-shu and YI Chang-lian from Beijing China, Wing Lau and S. Yang from Oxford University UK for their technical assistance.

\section{REFERENCES}

[1] G. Gregoire, G. Ryckewaert, L. Chevalier, et al, "MICE and International Muon Ionization Cooling Experiment Technical Reference Document," [Online]. Available: http://hep04.phys.itt.edu/cooldemo.

[2] D.R. Li, M. A. Green, S. P. Virostek, et al. "Progress on the RF Coupling Module for the MICE Channel," in Proceeding of the 2005 Particle Accelerator Conference, Knoxville TN, 2005, 2869-2871.

[3] L. Wang, M. A. Green, F. Y. Xu, et al, "The Engineering Design of the $1.5 \mathrm{~m}$ Diameter Solenoid for the MICE RFCC Modules", IEEE Transactions on Applied Superconductivity, Vol. 18, No. 2, 937-940, 2008.

[4] L. Wang, F. Y. Xu, H. Wu, et al, "Magnetic and Cryogenic Design of MICE Coupling Solenoid Magnet System,” IEEE Transactions on Applied Superconductivity, Vol. 19, No. 3, 1344-1347, June, 2009.

[5] Engineering design of MICE/MUCOOL coupling solenoid magnets, Institute of Cryogenics and Superconductivity Technology, Harbin Institute of Technology, 2008.

[6] E. S. Bobrov, J. E. C. Williams, and Y. Iwasa, "Experimental and theoretical investigation of mechanical disturbances coils. 2 . Shear-stress-induced epoxy fracture as the principal source of premature quenches and training-theoretical analysis," Cryogenics, Vol 25, pp 307-316, June 1985.

[7] M. Urata, and H. Maeda, "Relation between radial stress and quench current for tightly wound dry solenoids," IEEE Transactions on Magnetics, Vol. MAG-23, 1596-1599, March, 1987.

[8] S. G. Gong, and Y. Q. Huang. Finite Element Analysis and ANSYS APDL Programming and Its Application. Beijing, Machine Press, 2009, 192-200.

[9] ANSYS Theory Reference. ANSYS Inc., Pittsburgh, Penn., 2004.

[10] S. Yadav, and J. Hoffman, "Epoxies for Cryogenic Applications," Fermi Lab Note, TD-99-021, 1999. 